\title{
镍催化硅基保护烯丙醇的烯氢化反应：一种合成含 有手性季碳中心高烯丙醇的有效方法
}

\author{
张齐，朱守非，蔡艳，王立新，周其林 ${ }^{*}$ \\ 元素有机国家重点实验室; 南开大学元素有机化学研究所, 天津 300071 \\ *通讯作者, E-mail: qlzhou@nankai.edu.cn
}

收稿日期: 2010-05-01; 接受日期: 2010-06-01

\begin{abstract}
摘要报道了一例手性螺环亚磷酰胺配体和镍的络合物催化的硅基保护烯丙醇的不对称 烯氢化反应, 以较高的收率(最高达 $97 \%$ )和对映选择性(最高达 $95 \%$ ee)合成了一系列含有手 性季碳中心的高烯丙醇. 该反应为含有手性季碳中心的双官能团化合物的合成提供了新的 有效方法.

关键词

不对称烯氢化 手性螺环磷配体 官能团化烯烃 手性季碳中心
\end{abstract}

\section{1 引言}

过渡金属催化的碳-碳键形成反应是有机合成研 究的重点. 镍催化的不对称烯氢化反应使用工业原 料乙烯作为起始物合成有用的手性化合物, 是一类 原子经济性构筑碳-碳键的方法, 在精细化学品和制 药工业中有着重要应用价值 ${ }^{[1 \sim 3]}$. 在过去几十年里, 人们对该反应进行了大量的研究, 发展出几类高效 的手性镍催化剂, 实现了包括芳基乙烯 ${ }^{[4-8]} 、 \alpha$-烷基取 代的芳基乙烯 ${ }^{[9,10]}$ 、具有较大张力的双环烯烃 ${ }^{[11]}$ 和 1,3-二烯 ${ }^{[12,13]}$ 等非官能团化烯烃的对映选择性烯氢 化反应. 但是, 官能团化烯烃的烯氢化反应迄今仍然 是一个挑战性课题.

官能团化烯烃的烯氢化反应能够方便地合成多 官能团化产物. 由于多官能团化合物在生物活性化 合物合成中有广泛用途, 官能团化烯烃的烯氢化反 应有着重要意义. 最近我们发展了一例镍催化 $\alpha$-缩 酮取代芳基乙烯的烯氢化反应. 该反应能够方便地 合成烯丙基醛, 收率和化学选择性都很高 ${ }^{[14]}$. 当我们 使用手性螺环磷配体尝试该反应的不对称诱导时, 只得到较低的对映选择性 (24\% ee). 在随后的研究
中我们发现手性螺环亚磷酰胺配体在镍催化的烯丙 基硅醚的不对称烯氢化反应中具有很高的对映选择 性, 这是第一例高对映选择性的官能团化烯烃烯氢 化反应, 本文将对这一反应进行详细讨论.

\section{2 结果与讨论}

我们首先使用由 $2 \mathrm{~mol} \%[\mathrm{Ni} \text { (allyl)Br }]_{2}, 4 \mathrm{~mol} \%$ $\left(S_{a}, R, R\right.$ )-SIPHOS-PE，和 $5 \mathrm{~mol} \% \mathrm{NaBAr}_{\mathrm{F}}$ (四[3,5-二 (三氟甲基苯基)]硼酸钠)现场制备的催化剂, 以二氯 甲烷作为溶剂, 在室温下考察了二甲基叔丁基硅基 保护的 2-苯基烯丙醇 $1 \mathrm{a}$ 的烯氢化反应. 上述烯氢化 反应能够顺利发生，以较高的收率 (69\%) 和中等对 映选择性 (41\%) 得到稀氢化产物 (式 1). 该反应存 在一个主要的竞争副反应, 即原料 $1 \mathrm{a}$ 中的双键发生 迁移, 得到副产物 3a. 这种副反应在镍催化烯氢化 反应中经常遇到.

在前期的研究中我们曾经发现, 烯烃底物中官 能团的配位能力对镍催化烯氢化反应的活性和选择 性都有较大影响 ${ }^{[14]}$. 具有较强配位能力的官能团占 据了镍催化剂的一个配位点, 阻止了烯烃与镍的配 


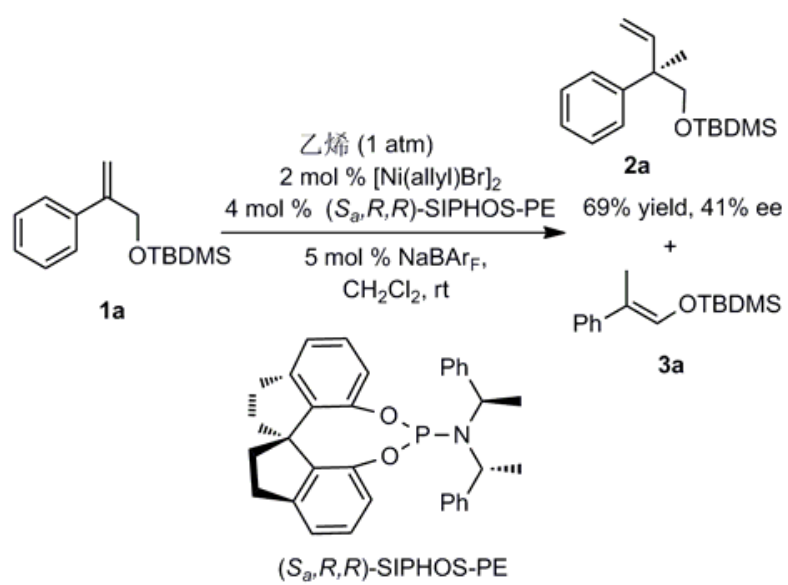

式 1 镍催化二甲基叔丁基(2-苯基烯丙氧基)硅烷 $1 \mathbf{a}$ 的不对 称烯氢化反应. 式中 TBDMS 表示二甲基叔丁基硅基

位，进而阻碍烯氢化反应的进行. 因而我们相信，在 镍催化官能团化烯烃的不对称烯氢化反应中，烯烃 底物官能团保护基的正确选择对获得高反应活性、高 化学选择性、以及高对映选择性都至关重要. 基于这 种考虑, 我们首先考察了具有不同保护基的烯丙醇 底物对烯氢化反应的影响, 结果如表 1 所示. 从表 1 可以看出, 未经保护的烯丙醇反应活性很低, 而且产 物成分复杂 (实验 2). 当引入硅保护基时, 相应烯氢 化反应能够顺利进行 (实验 1,3 和 4). 和二甲基叔丁 基硅基 (TBDMS, 底物 1a) 相比, 体积较小的三甲 基硅基 (TMS, 底物 1c) 保护时, 反应的对映选择性 有所提高（实验 1 和 3); 而使用更大体积的三异丙基 硅基保护基 (TIPS, 底物 1d) 时, 反应的收率和对映 选择性都有明显降低 (实验 4). 除了硅基保护基外,

表 1 镍催化烯丙醇的不对称烯氢化反应: 保护基比较 ${ }^{\text {a) }}$

\begin{tabular}{|c|c|c|c|c|}
\hline 实验 & 底物 & $\mathrm{PG}$ & $\mathbf{4 c}$ 的收率 $(\%)^{\mathrm{b})}$ & $4 \mathbf{c}$ 的 ee $(\%)^{c)}$ \\
\hline 1 & $1 \mathrm{a}$ & TBDMS & 69 & 41 \\
\hline 2 & $1 b$ & $\mathrm{H}$ & $\mathrm{ND}^{\mathrm{d})}$ & - \\
\hline 3 & 1c & TMS & 56 & 60 \\
\hline 4 & 1d & TIPS & 31 & 25 \\
\hline 5 & $1 e$ & MOM & 49 & -22 \\
\hline 6 & 1f & $\mathrm{Bn}$ & $35^{\mathrm{e})}$ & $20^{\mathrm{e})}$ \\
\hline
\end{tabular}

a) 反应条件: $[\mathrm{Ni}(\mathrm{allyl}) \mathrm{Br}]_{2}(0.01 \mathrm{mmol}),\left(S_{a}, R, R\right)$-SIPHOS-PE (0.02 mmol), NaBAr $(0.024 \mathrm{mmol}), \mathbf{1}(0.5 \mathrm{mmol}), \mathrm{CH}_{2} \mathrm{Cl}_{2}(2.5 \mathrm{~mL}), \mathrm{rt}$, 1 3 h. b) 两步总收率. c) 用 SFC (超临界流体色谱) 测定. 手性色谱 柱为 Chiralcel OJ-H. d) 未检测. e) 为脱保护产物 $\mathbf{5}$ 的数据.
我们还考察了甲氧甲基 (MOM) 和苠基 (Bn) 保护 的烯丙醇 $1 \mathrm{e}$ 和 $\mathbf{1 f}$ 的烯氢化反应. 在标准反应条件下, 这两种底物反应的收率和对映选择性都较低 (实验 5 和 6).

为了进一步提高反应的对映选择性, 我们对反 应条件进行了系统优化 (表 2). 首先我们比较了不同 的溶剂, 结果发现反应在二氯甲烷中给出最好结果 (实验 2 5). 降低温度对提高反应对映选择性有利, 当反应温度从室温逐渐降低到 $0{ }^{\circ} \mathrm{C},-40{ }^{\circ} \mathrm{C}$ 和 $-60{ }^{\circ} \mathrm{C}$ 时, 反应对映选择性逐步从 $60 \%$ ee 提高到 $81 \%$ ee (实验6 8). 温度降低时, 反应活性有所降低, 但是反 应仍能在 $5 \mathrm{~h}$ 以内结束. 由于低温下烯氢化反应选择 性提高, 所以目标产物的收率也从 $56 \%$ 提高到 $77 \%$ (实验 1 和 8 ).

在最优反应条件下, 我们比较了各种手性螺环 单磷配体 ${ }^{[15]}$ (图 1) 在镍催化硅基保护烯丙醇 $1 \mathrm{c}$ 的不 对称烯氢化反应中手性诱导效果, 发现含手性胺结 构的螺环单齿亚磷酰胺配体 $\left(S_{a}, R, R\right)$-SIPHOS-PE 在 反应中给出最好的收率和对映选择性, 其余配体的 活性和对映选择性都不理想.

接下来, 我们对反应的底物适用范围进行了 考查 (表 3). 芳环上带有间位或者对位取代的三甲 基 (2-芳基烯丙氧基) 硅烷都能在 $\mathrm{Ni}-\left(S_{a}, R, R\right)$ SIPHOS-PE 催化下顺利完成不对称烯氢化反应, 以 中等或较高的收率 (42\% 97\%) 和很高的对映选择 性 (76\% 95\% ee)生成相应的含有手性季碳中心的高

表 2 镍催化三甲基(2-苯基烯丙氧基)硅烷的不对称烯氢化 反应：反应条件优化 ${ }^{\text {a) }}$



a) 反应条件以及分析方法和表 1 , 实验 3 的相同. b) 通过 GC 测定. 色谱柱为 HP-5. 


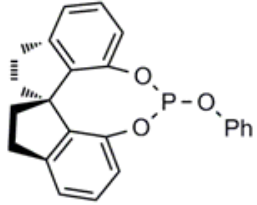

(S)-ShiP

$31 \%, 3 \%$ ee

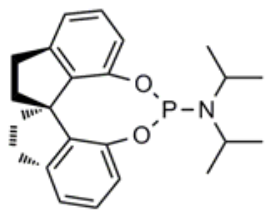

(R)-SIPHOS-'Pr $69 \%,-42 \%$ ee

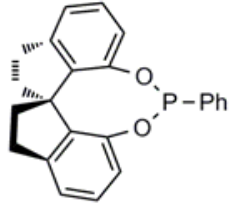

(S)-FuP

$<10 \%$ conv

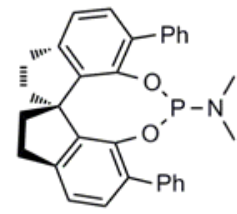

(S)-6,6'-diPh-SIPHOS $<10 \%$ conv.

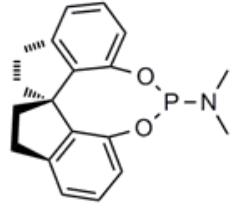

(S)-SIPHOS $43 \%, 30 \%$ ee
图 1 配体比较 (采用和表 2, 实验 8 相同的反应条件)

表 3 镍催化三甲基(2-芳基烯丙氧基)硅烷的不对称烯氢化 反应 ${ }^{\text {a) }}$

\begin{tabular}{|c|c|c|c|c|c|}
\hline 1 & $\bigcap_{\text {ОтмS }} \frac{4}{2}$ & $\begin{array}{l}\text { 己烯 (1 atm) } \\
2 \mathrm{~mol} \% \text { [Ni(allyl)Br } \\
\left.\text { ol \%( } \mathrm{S}_{a}, R, R\right)-\mathrm{SIPHC} \\
5 \mathrm{~mol}^{2} \mathrm{NaBAr}_{\mathrm{F}}, \\
\mathrm{CH}_{2} \mathrm{Cl}_{2},-60^{\circ} \mathrm{C}\end{array}$ & & $\underset{\mathrm{THF}}{\stackrel{\mathrm{TBAF}}{\longrightarrow}}$ & $\mathrm{OH}$ \\
\hline 实验 & 底物 & $\mathrm{Ar}$ & 产物 & 收率 (\%) & ee $(\%)$ \\
\hline 1 & $1 c$ & $\mathrm{C}_{6} \mathrm{H}_{5}$ & $4 c$ & 77 & $81(R)$ \\
\hline $2^{\mathrm{b})}$ & $1 \mathrm{~g}$ & 4- $\mathrm{MeC}_{6} \mathrm{H}_{4}$ & $4 g$ & 91 & $78(R)$ \\
\hline 3 & $1 \mathrm{~h}$ & 4- $\mathrm{MeOC}_{6} \mathrm{H}_{4}$ & $4 h$ & 97 & 85 \\
\hline 4 & 1i & 4- $-\mathrm{FC}_{6} \mathrm{H}_{4}$ & $4 \mathbf{i}$ & 80 & 94 \\
\hline 5 & $\mathbf{1 j}$ & $4-\mathrm{ClC}_{6} \mathrm{H}_{4}$ & $4 \mathbf{j}$ & 69 & 92 \\
\hline 6 & $1 k$ & $4-\mathrm{BrC}_{6} \mathrm{H}_{4}$ & $4 k$ & 62 & 95 \\
\hline 7 & 11 & 4- $\mathrm{PhC}_{6} \mathrm{H}_{4}$ & 41 & 82 & 80 \\
\hline 8 & $1 \mathrm{~m}$ & $3-\mathrm{MeOC}_{6} \mathrm{H}_{4}$ & $4 m$ & 86 & 76 \\
\hline 9 & 1n & $3-\mathrm{FC}_{6} \mathrm{H}_{4}$ & $4 n$ & 49 & 91 \\
\hline 10 & 10 & $3-\mathrm{ClC}_{6} \mathrm{H}_{4}$ & 40 & 42 & 88 \\
\hline 11 & $1 p$ & $3-\mathrm{BrC}_{6} \mathrm{H}_{4}$ & $4 p$ & 49 & 85 \\
\hline 12 & $1 q$ & & $4 q$ & 92 & 89 \\
\hline 13 & $1 \mathbf{r}$ & 2-naphthyl & $4 r$ & 92 & 81 \\
\hline 14 & $1 \mathrm{~s}$ & & $4 s$ & 90 & 73 \\
\hline
\end{tabular}

a) 反应条件及分析方法和表 2 中实验 8 的相同. b) 反应在 $-40{ }^{\circ} \mathrm{C}$ 下进行.

烯丙醇产物 (实验 1 12). 苯环对位含有给电子取代 基的底物 $1 \mathrm{~g}$ 和 $1 \mathrm{~h}$ 给出很高的收率 (分别为 $91 \%$ 和 $97 \%$ ), 但对映选择性相对较低 (分别为 $78 \%$ ee 和 $85 \%$ ee) (实验 2 和 3). 相反, 苯环对位含有拉电子取 代基的底物 1i, 1j 和 1k 给出较低的收率（62\% 80\%),
但对映选择性很高（92\% 95\% ee）（实验 4 6). 苯 环对位苯基取代的底物 11 也能给出较高的收率和对 映选择性 (实验 7). 苯环上间位含有取代基的烯丙 醇硅醚底物普遍给出较低的收率, 其对映选择性也 比相应对位取代底物的结果差 (对照实验 8 11 与实 验 3 6). 除了单取代苯基, 含双取代苯基的底物 1q 也能很好地进行相应烯氢化产物 (实验 12). 该催化 体系对底物的邻位取代较为敏感, 苯环邻位有取代 时, 烯氢化反应在标准条件下不能发生. 荎基和噻 吩基取代的烯丙醇硅醚 1r 和 $1 \mathrm{~s}$ 也能进行相应的烯 氢化反应, 反应的收率和对映选择性都较高（实验 13 和 14).

镍催化不对称烯氢化反应能够合成含有手性季 碳中心的高烯丙醇. 由于产物中含有碳-碳双键和羟 基官能团, 因此可以方便地转化为很多在合成上有 重要用途的双官能团化合物. 例如, 产物 $(S)-\mathbf{4 c}$ 通过 臭氧和双氧水氧化得到 $(S)$ - $\alpha$-甲基莨宕酸 (6) (式 2). $(S)-\alpha$-甲基莨宕酸是合成手性药物 $(S)$-阿托美品的关 键中间体 ${ }^{[16]}$.

\section{3 结论}

本文报道了一例镍催化官能团化烯烃的高对映 选择性烯氢化反应. 镍和手性螺环亚磷酰胺配体的 络合物能够催化烯丙醇硅醚的不对称烯氢化反应, 以较高的收率和对映选择性得到含有手性季碳中心 的高烯丙醇产物. 反应为含有手性季碳中心的双官 能团化合物的合成提供了新的有效方法.

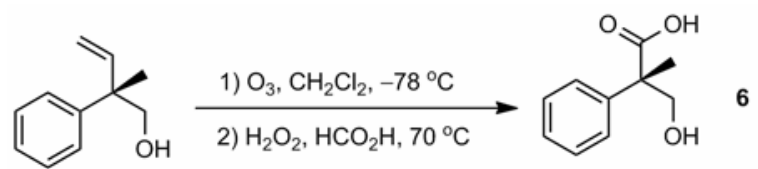

$(S)-4 c$ $[\alpha]_{D}^{20}=-19.0\left(\mathrm{c} 1.0, \mathrm{CHCl}_{3}\right)$
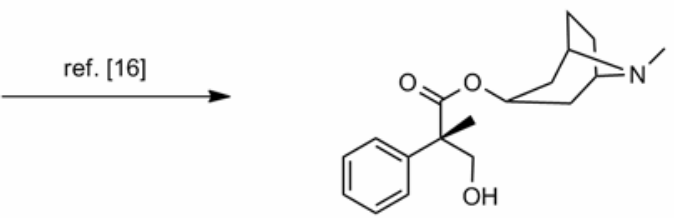

(S)-atromepine

式 2 (S)-阿托美品的关键中间体 $(S)$ - $\alpha$-甲基莨宕酸的不对称 合成 


\section{4 实验部分}

\section{1 仪器和试剂}

熔点用天津分析仪器厂 RY-1 型熔点仪测定, 温 度计未校正. ${ }^{1} \mathrm{H}$ NMR、 ${ }^{13} \mathrm{C}$ NMR、 ${ }^{31} \mathrm{P}$ NMR 核磁共振 谱由 Bruker $300 \mathrm{M}$ 核磁共振仪和 Varian $400 \mathrm{M}$ 核磁 共振仪测定. HRMS 由 APEXII 型 FT-ICR 质谱仪和 ZAB-HS 双聚焦高分辨有机质谱仪测定. 比旋光度由 Perkin Elmer $341 \mathrm{MC}$ 旋光仪测定. 所有的空气敏感 化合物均在 DRI-LAB HE493 或者 MBRAUN Lab Star 手套箱中称量并在真空线上按照标准 Schlenk 技术 (SST)操作. SFC 由 Mettler-Toledo 超临界流体色谱完 成. HPLC 分析由 Hewlett Packard Model HP 1100 高 效液相色谱仪或 Waters 2996 高效液相色谱仪完成. GC 分析由 Hewlett Packard Model HP 6890 气谱仪完 成. 臭氧发生器为 Ozone Services Yanco industries LTD.

二氯甲烷，1,2-二氯乙烷 (DCE), 氯仿经氢化钲 回流干燥, 重蒸; 乙醚, 四氢呋喃 (THF), 甲苯经钠二苯甲酮回流干燥, 重蒸. 其他溶剂如无特殊说明为 直接使用. 所有试剂均从 Aldrich, Acros 或 Alfa Asar 公司及国内的试剂公司购买. $\left[\mathrm{Ni}(\text { allyl)Br }]_{2}{ }^{[17]}\right.$, $\mathrm{NaBAr}_{\mathrm{F}}{ }^{[18,19]}$ 手性螺环单磷配体 ${ }^{[20 ~ 22]}$ 均参考文献方法 合成. 其中 ShiP, SIPHOS, 和 SIPHOS-PE 均可从 Aldrich 或 Strem 试剂公司购买. 2-芳基丙-2-烯-1-醇 按照文献方法 ${ }^{[23]}$, 在催化量 $\mathrm{CuI}$ 存在下, 芳基溴化镁 与丙-2-炔-1-醇反应制得.

\section{2 底物合成}

制备三甲基(2-芳基烯丙氧基)硅烷的典型操作: 向 $100 \mathrm{~mL}$ 双口瓶中加入 2-芳基烯丙醇 (10 mmol), 吡啶 $(11 \mathrm{mmol})$ 和干燥的 THF $(50 \mathrm{~mL})$, 然后滴加三 甲基氯硅烷 (10 mmol), 室温搅拌 $1 \mathrm{~h}$, TLC 显示原料 消失. 将体系过滤, 滤液浓缩后经硅胶柱层析 (石油 醚 $/$ 乙酸乙酯 $=10: 1, v / v)$ 提纯得到目标产物, 其分 析数据如下.

三甲基(2-苯基烯丙氧基)硅烷 (1c)

无色液体, 收率 $92 \%$ (从丙-2-炔-1-醇为起始原 料算起). ${ }^{1} \mathrm{H}$ NMR (400 MHz, $\left.\mathrm{CDCl}_{3}\right) \delta 7.43 \sim 7.26(\mathrm{~m}$, $5 \mathrm{H}), 5.45(\mathrm{~s}, 1 \mathrm{H}), 5.39(\mathrm{~s}, 1 \mathrm{H}), 4.52(\mathrm{~s}, 2 \mathrm{H}), 0.17(\mathrm{~s}$, $9 \mathrm{H}) ;{ }^{13} \mathrm{C}$ NMR $\left(100 \mathrm{MHz}, \mathrm{CDCl}_{3}\right) \delta 147.1,139.3,128.6$, 127.9, 126.3, 112.1, 64.6, 0.2; HRMS (EI) 计算值
$\mathrm{C}_{12} \mathrm{H}_{18} \mathrm{OSi}: 206.1127$; 实测值 206.1144.

三甲基(2-对甲苯基烯丙氧基)硅烷 (1g)

无色液体，收率 68\% (从丙-2-炔-1-醇为起始原 料算起). ${ }^{1} \mathrm{H}$ NMR $\left(300 \mathrm{MHz}, \mathrm{CDCl}_{3}\right) \delta 7.16(\mathrm{~d}, J=8.1$ $\mathrm{Hz}, 2 \mathrm{H}), 6.99$ (d, J = 7.5 Hz, 2H), 5.25 (s, 1H), 5.18 (s, $1 \mathrm{H}), 4.34(\mathrm{~s}, 2 \mathrm{H}), 2.19(\mathrm{~s}, 3 \mathrm{H}), 0.00(\mathrm{~s}, 9 \mathrm{H}) ;{ }^{13} \mathrm{C} \mathrm{NMR}$ $\left(100 \mathrm{MHz}, \mathrm{CDCl}_{3}\right) \delta 146.9,137.6,136.4,129.3,126.2$, 111.3, 64.6, 21.4, -0.1; HRMS (EI) 计算值 $\mathrm{C}_{13} \mathrm{H}_{20} \mathrm{OSi}$ : 220.1283 ; 实测值 220.1284 .

三甲基(2-(4-甲氧基苯基)烯丙氧基)硅烷 (1h)

无色液体, 收率 29\% (从丙-2-炔-1-醇为起始原 料算起). ${ }^{1} \mathrm{H}$ NMR (300 MHz, $\left.\mathrm{CDCl}_{3}\right) \delta 7.21$ (d, $J=8.7$ $\mathrm{Hz}, 2 \mathrm{H}), 6.72(\mathrm{~d}, J=8.7 \mathrm{~Hz}, 2 \mathrm{H}), 5.21(\mathrm{~s}, 1 \mathrm{H}), 5.13$ (s, $1 \mathrm{H}), 4.33$ (s, 2H), $3.66(\mathrm{~s}, 3 \mathrm{H}), 0.00(\mathrm{~s}, 9 \mathrm{H}) ;{ }^{13} \mathrm{C} \mathrm{NMR}$ $\left(75 \mathrm{MHz}, \mathrm{CDCl}_{3}\right) \delta 159.7,146.6,131.9,127.5,114.1$, $110.8,64.9,55.5,0.0$; HRMS (EI) 计算值 $\mathrm{C}_{13} \mathrm{H}_{20} \mathrm{O}_{2} \mathrm{Si}$ : 236.1233 ; 实测值 236.1237 .

三甲基(2-(4-氟苯基)烯丙氧基)硅烷 (1i)

无色液体, 收率 $60 \%$ (从丙-2-炔-1-醇为起始原 料算起). ${ }^{1} \mathrm{H} \mathrm{NMR}\left(300 \mathrm{MHz}, \mathrm{CDCl}_{3}\right) \delta 7.43$ (dd, $J=$ 8.8 and $5.6 \mathrm{~Hz}, 2 \mathrm{H}), 7.04(\mathrm{t}, J=8.8 \mathrm{~Hz}, 2 \mathrm{H}), 5.43(\mathrm{~s}$, $1 \mathrm{H}), 5.42(\mathrm{~s}, 1 \mathrm{H}), 4.53(\mathrm{~s}, 2 \mathrm{H}), 0.22(\mathrm{~s}, 9 \mathrm{H}) ;{ }^{13} \mathrm{C} \mathrm{NMR}$ $\left(100 \mathrm{MHz}, \mathrm{CDCl}_{3}\right) \delta 163.9,161.4,146.2,135.3,128.0$, $127.9,115.4,115.2,112.2,64.7,-0.3$; HRMS (EI) 计 算值 $\mathrm{C}_{12} \mathrm{H}_{17} \mathrm{FOSi}$ : 224.1033; 实测值 224.1032.

三甲基(2-(4-氯苯基)烯丙氧基)硅烷 (1j)

无色液体，收率 34\%(从丙-2-炔-1-醇为起始原料 算起). ${ }^{1} \mathrm{H} \mathrm{NMR}\left(300 \mathrm{MHz}, \mathrm{CDCl}_{3}\right) \delta 7.22 \sim 7.13$ (m, 4H), $5.28(\mathrm{~s}, 1 \mathrm{H}), 5.23(\mathrm{~s}, 1 \mathrm{H}), 4.32(\mathrm{~s}, 2 \mathrm{H}), 0.00(\mathrm{~s}$, $9 \mathrm{H}) ;{ }^{13} \mathrm{C}$ NMR $\left(100 \mathrm{MHz}, \mathrm{CDCl}_{3}\right) \delta 146.0,137.6,133.7$, 128.6, 127.6, 112.9, 64.5, -0.2; HRMS (EI) 计算值 $\mathrm{C}_{12} \mathrm{H}_{17} \mathrm{ClOSi}$ : 240.0737; 实测值 240.0741.

三甲基(2-(4-溴苯基)烯丙氧基)硅烷 (1k)

无色液体, 收率 47\% (从丙-2-炔-1-醇为起始原 料算起). ${ }^{1} \mathrm{H}$ NMR (400 MHz, $\left.\mathrm{CDCl}_{3}\right) \delta 7.45$ (d, $J=8.4$ $\mathrm{Hz}, 2 \mathrm{H}), 7.30$ (d, $J=8.4 \mathrm{~Hz}, 2 \mathrm{H}), 5.45$ (s, 1H), 5.40 (s, $1 \mathrm{H}), 4.48$ (s, 2H), 0.17 (s, 9H); ${ }^{13} \mathrm{C}$ NMR (100 MHz, $\left.\mathrm{CDCl}_{3}\right) \delta 146.1,139.1,131.6,128.0,121.9,113.0,64.5$, -0.2 ; HRMS (EI) 计算值 $\mathrm{C}_{12} \mathrm{H}_{17} \mathrm{BrOSi}$ : 284.0232; 实 测值 284.0235 .

三甲基(2-(4-联苯基)烯丙氧基)硅烷 (11)

白色固体 $\left(\mathrm{mp}: 58 \sim 60{ }^{\circ} \mathrm{C}\right.$ ), 收率 $41 \%$ (从丙-2炔-1-醇为起始原料算起). ${ }^{1} \mathrm{H} \mathrm{NMR}\left(300 \mathrm{MHz}, \mathrm{CDCl}_{3}\right)$ 
$\delta 7.59-7.30(\mathrm{~m}, 9 \mathrm{H}), 5.52(\mathrm{~s}, 1 \mathrm{H}), 5.44(\mathrm{~s}, 1 \mathrm{H}), 4.56(\mathrm{~s}$, $2 \mathrm{H}), 0.20(\mathrm{~s}, 9 \mathrm{H}) ;{ }^{13} \mathrm{C} \mathrm{NMR}\left(75 \mathrm{MHz}, \mathrm{CDCl}_{3}\right) \delta 146.6$, 141.0, 140.8, 138.2, 129.1, 127.7, 127.3, 127.2, 126.8, 112.3，64.7，0.0; HRMS (EI) 计算值 $\mathrm{C}_{18} \mathrm{H}_{22} \mathrm{OSi}$ : 282.1440; 实测值 282.1433 .

三甲基(2-(3-甲氧基苯基)烯丙氧基)硅烷 (1m)

无色液体, 收率 34\% (从丙-2-炔-1-醇为起始原 料算起). ${ }^{1} \mathrm{H}$ NMR $\left(300 \mathrm{MHz}, \mathrm{CDCl}_{3}\right) \delta 7.35 \sim 6.90(\mathrm{~m}$, $4 \mathrm{H}), 5.55(\mathrm{~s}, 1 \mathrm{H}), 5.50(\mathrm{~s}, 1 \mathrm{H}), 4.60(\mathrm{~s}, 2 \mathrm{H}), 3.86(\mathrm{~s}$, $3 \mathrm{H}), 0.28(\mathrm{~s}, 9 \mathrm{H}) ;{ }^{13} \mathrm{C}$ NMR $\left(75 \mathrm{MHz}, \mathrm{CDCl}_{3}\right) \delta 160.1$, 147.3, 141.1, 129.7, 119.0, 113.4, 112.6, 112.5, 64.8, 55.5, 0.0; HRMS (EI) 计算值 $\mathrm{C}_{13} \mathrm{H}_{20} \mathrm{O}_{2} \mathrm{Si}$ : 236.1233; 实测值 236.1234 .

三甲基(2-(3-氟苯基)烯丙氧基)硅烷 (1n)

无色液体, 收率 56\% (从丙-2-炔-1-醇为起始原 料算起). ${ }^{1} \mathrm{H}$ NMR (300 MHz, $\left.\mathrm{CDCl}_{3}\right) \delta 7.12 \sim 6.75(\mathrm{~m}$, 4H), $5.29(\mathrm{~s}, 1 \mathrm{H}), 5.25(\mathrm{~s}, 1 \mathrm{H}), 4.31(\mathrm{~s}, 2 \mathrm{H}), 0.00(\mathrm{~s}$, 9H) $;{ }^{13} \mathrm{C}$ NMR $\left(75 \mathrm{MHz}, \mathrm{CDCl}_{3}\right) \delta 165.1,161.8,146.4$, 142.0, 141.9, 130.3, 130.2, 122.2, 115.1, 114.8, 113.7, 113.5, 64.7, 0.0; HRMS (EI) 计算值 $\mathrm{C}_{12} \mathrm{H}_{17} \mathrm{FOSi}$ : 224.1033; 实测值 224.1034.

三甲基(2-(3-氯苯基)烯丙氧基)硅烷 (1o)

无色液体, 收率 49\% (从丙-2-炔-1-醇为起始原 料算起). ${ }^{1} \mathrm{H}$ NMR (400 MHz, $\left.\mathrm{CDCl}_{3}\right) \delta 7.41 \sim 7.26(\mathrm{~m}$, 4H), $5.47(\mathrm{~s}, 1 \mathrm{H}), 5.42(\mathrm{~s}, 1 \mathrm{H}), 4.78(\mathrm{~s}, 2 \mathrm{H}), 0.17(\mathrm{~s}$, 9H) $;{ }^{13} \mathrm{C}$ NMR (75 MHz, $\left.\mathrm{CDCl}_{3}\right) \delta 146.2,141.4,134.7$, 130.0, 128.1, 126.7, 124.7, 113.6, 64.7, 0.0; HRMS (EI) 计算值 $\mathrm{C}_{12} \mathrm{H}_{17} \mathrm{ClOSi}$ : 240.0737; 实测值 240.0738.

三甲基(2-(3-溴苯基)烯丙氧基)硅烷 (1p)

无色液体, 收率 33\% (从丙-2-炔-1-醇为起始原 料算起). ${ }^{1} \mathrm{H}$ NMR (300 MHz, $\left.\mathrm{CDCl}_{3}\right) \delta 7.40 \sim 6.99(\mathrm{~m}$, $4 \mathrm{H}), 5.28(\mathrm{~s}, 1 \mathrm{H}), 5.24(\mathrm{~s}, 1 \mathrm{H}), 4.30(\mathrm{~s}, 2 \mathrm{H}), 0.00(\mathrm{~s}$, 9H); ${ }^{13} \mathrm{C}$ NMR (75 MHz, $\left.\mathrm{CDCl}_{3}\right) \delta 146.1,141.6,131.0$, 130.2, 129.6, 125.1, 123.0, 113.7, 64.6, 0.0; HRMS (EI) 计算值 $\mathrm{C}_{12} \mathrm{H}_{17} \mathrm{BrOSi}$ : 284.0232; 实测值 284.0234.

三甲基(2-(苯并 $[1,3]$ 二氧环戊烷-5-基)烯丙氧基) 硅烷 $\mathbf{( 1 q )}$

无色液体, 收率 32\%(从丙-2-炔-1-醇为起始原料 算起). ${ }^{1} \mathrm{H}$ NMR $\left(300 \mathrm{MHz}, \mathrm{CDCl}_{3}\right) \delta 6.78 \sim 6.57$ (m, $3 \mathrm{H}), 5.74(\mathrm{~s}, 2 \mathrm{H}), 5.18(\mathrm{~s}, 1 \mathrm{H}), 5.13(\mathrm{~s}, 1 \mathrm{H}), 4.28(\mathrm{~s}$, $2 \mathrm{H}), 0.00(\mathrm{~s}, 9 \mathrm{H}) ;{ }^{13} \mathrm{C} \mathrm{NMR}\left(75 \mathrm{MHz}, \mathrm{CDCl}_{3}\right) \delta 148.1$, 147.6, 146.7, 133.7, 119.9, 111.5, 108.4, 107.1, 101.4, 65.0, 0.0; HRMS (EI) 计算值 $\mathrm{C}_{13} \mathrm{H}_{18} \mathrm{O}_{3} \mathrm{Si}$ : 250.1025; 实
测值 250.1058 .

三甲基(2-(2-荎基)烯丙氧基)硅烷 (1r)

黄色液体, 收率 74\% (从丙-2-炔-1-醇为起始原 料算起). ${ }^{1} \mathrm{H}$ NMR $\left(300 \mathrm{MHz}, \mathrm{CDCl}_{3}\right) \delta 7.83 \sim 7.39(\mathrm{~m}$, $7 \mathrm{H}), 5.61(\mathrm{~s}, 1 \mathrm{H}), 5.51(\mathrm{~s}, 1 \mathrm{H}), 4.64(\mathrm{~s}, 2 \mathrm{H}), 0.20(\mathrm{~s}$, 9H); ${ }^{13} \mathrm{C}$ NMR $\left(75 \mathrm{MHz}, \mathrm{CDCl}_{3}\right) \delta 146.9,136.5,133.7$, 133.3, 128.5, 128.2, 127.9, 126.5, 126.2, 124.9, 124.8, $112.9,64.8,0.0 ;$ HRMS (EI) 计算值 $\mathrm{C}_{16} \mathrm{H}_{20} \mathrm{OSi}$ : 256.1283; 实测值 256.1290 .

三甲基(2-(2-噻吩基)烯丙氧基)硅烷 (1s)

无色液体, 收率 32\% (从丙-2-炔-1-醇为起始原 料算起). ${ }^{1} \mathrm{H}$ NMR $\left(300 \mathrm{MHz}, \mathrm{CDCl}_{3}\right) \delta 7.04 \sim 6.84(\mathrm{~m}$, $3 \mathrm{H}), 5.39(\mathrm{~s}, 1 \mathrm{H}), 5.18(\mathrm{~s}, 1 \mathrm{H}), 4.37(\mathrm{~s}, 2 \mathrm{H}), 0.08(\mathrm{~s}$, 9H) ${ }^{13} \mathrm{C}$ NMR (75 MHz, $\mathrm{CDCl}_{3}$ ) $\delta 142.7,141.0,127.6$, 124.6，123.6，111.0，64.7，0.0; HRMS (EI) 计算值 $\mathrm{C}_{10} \mathrm{H}_{16} \mathrm{OSSi}$ : 212.0691; 实测值 212.0697.

二甲基叔丁基(2-苯基烯丙氧基)硅烷 (1a)

氮气保护下, 向 $100 \mathrm{~mL}$ 二口瓶中加入 2-苯基烯 丙醇 $(2.4 \mathrm{~g}, 18 \mathrm{mmol})$, 咪唑 $(13.6 \mathrm{~g}, 203 \mathrm{mmol})$, 叔 丁基二甲基氯硅烷 $(3.0 \mathrm{~g}, 20 \mathrm{mmol})$ 和干燥的 DMF $(40 \mathrm{~mL})$. 室温摚拌 $20 \mathrm{~h}$, TLC 显示原料消失, 将体系 倒入 $200 \mathrm{~mL}$ 水中, 加入 $80 \mathrm{~mL} \mathrm{CH}_{2} \mathrm{Cl}_{2}$, 分液, 并用 2 $\times 50 \mathrm{~mL} \mathrm{CH} \mathrm{Cl}_{2}$ 萃取水相. 合并有机相, 并用冷的 $1 \mathrm{M} \mathrm{HCl}$ 溶液、饱和 $\mathrm{NaHCO}_{3}$ 溶液和饱和氯化钠溶液 洗涤有机相各一次, 无水硫酸钠干燥. 浓缩后硅胶柱 层析(石油梄)乙酸乙酯 $=10: 1, v / v$ ), 得 $4.2 \mathrm{~g}$ 二甲基 叔丁基(2-苯基烯丙氧基)硅烷 1a, 收率 $95 \%$, 为无色 油状液体. ${ }^{1} \mathrm{H}$ NMR $\left(400 \mathrm{MHz}, \mathrm{CDCl}_{3}\right) \delta 7.42 \sim 7.26$ $(\mathrm{m}, 5 \mathrm{H}), 5.43(\mathrm{~d}, J=1.6 \mathrm{~Hz}, 1 \mathrm{H}), 5.41(\mathrm{~d}, J=2.0 \mathrm{~Hz}$, 1H), 4.54 (s, 2H), 0.95 (s, 9H), 0.12 (s, 6H); ${ }^{13} \mathrm{C}$ NMR $\left(75 \mathrm{MHz}, \mathrm{CDCl}_{3}\right) \delta 147.2,139.3,128.4,127.8,126.2$, $111.5 ， 64.9 ， 26.1 ， 18.5 ， 0.0$; HRMS (EI) 计算值 $\mathrm{C}_{11} \mathrm{H}_{15} \mathrm{OSi}\left(\mathrm{M}-\mathrm{C}_{4} \mathrm{H}_{9}\right)$ : 191.0892; 实测值 191.0897.

三异丙基(2-苯基烯丙氧基)硅烷 (1d)

化合物 1d 采用与 $1 \mathrm{a}$ 相同的方法, 以 2-苯基烯丙 醇和三异丙基氯硅烷为起始原料合成. 收率 $98 \%$, 为 浅黄色油状物。 ${ }^{1} \mathrm{H}$ NMR (400 $\left.\mathrm{MHz}, \mathrm{CDCl}_{3}\right) \delta$ 7.40 7.28 (m, 5H), $5.49(\mathrm{~s}, 1 \mathrm{H}), 5.44(\mathrm{~s}, 1 \mathrm{H}), 4.61(\mathrm{~s}$, 2H), 1.20 1.09 (m, 21H); ${ }^{13} \mathrm{C}$ NMR (75 MHz, $\left.\mathrm{CDCl}_{3}\right) \delta$ 147.1, 139.3, 128.4, 127.7, 126.0, 111.1, 64.9, 18.2, 12.2; HRMS (EI) 计算值 $\mathrm{C}_{15} \mathrm{H}_{23} \mathrm{OSi} \quad\left(\mathrm{M}-\mathrm{C}_{3} \mathrm{H}_{7}\right)$ : 247.1518; 实测值 247.1513.

\section{2-苯基烯丙醇甲氧基甲醚（1e)}


氮气保护下, 向 $100 \mathrm{~mL}$ 双口瓶中加入 2-苯基烯 丙醇 $(1.9 \mathrm{~g}, 14 \mathrm{mmol}), \mathrm{NaH}(0.36 \mathrm{~g}, 15 \mathrm{mmol})$ 和干 燥的 THF $(50 \mathrm{~mL})$. 室温摚拌 $4 \mathrm{~h}$, 溶液呈棕色. 此时 将 $\mathrm{MeOCH}_{2} \mathrm{Cl}(1.4 \mathrm{~g}, 17 \mathrm{mmol})$ 逐滴滴入其中, 室温 摚拌过夜. TLC 显示原料消失后, 将反应液倒入约 $100 \mathrm{~mL}$ 水中, 用乙醚萃取 $(80 \times 2 \mathrm{~mL})$, 用饱和氯化 钠溶液洗有机相一次, 无水硫酸钠干燥. 浓缩后硅胶 柱层析 (石油醚/乙酸乙酯 $=10: 1, v / v$ ), 得 $2.1 \mathrm{~g} 2$-苯 基烯丙醇甲氧基甲醚 1e, 收率 85\%, 为无色液体. ${ }^{1} \mathrm{H}$ NMR $\left(400 \mathrm{MHz}, \mathrm{CDCl}_{3}\right) \delta 7.47(\mathrm{~d}, J=6.8 \mathrm{~Hz}, 2 \mathrm{H})$, $7.36 \sim 7.29(\mathrm{~m}, 3 \mathrm{H}), 5.54(\mathrm{~s}, 1 \mathrm{H}), 5.37(\mathrm{~s}, 1 \mathrm{H}), 4.70(\mathrm{~s}$, 2H), 4.47 (s, 2H), 3.38 (s, 3H); ${ }^{13} \mathrm{C} \mathrm{NMR}\left(\mathrm{CDCl}_{3}, 75\right.$ MHz) $\delta 144.2,138.9,128.4,127.8,126.1,114.3,95.5$, 68.9, 55.3; HRMS (EI) 计算值 $\mathrm{C}_{11} \mathrm{H}_{14} \mathrm{O}_{2}$ : 178.0994; 实测值 178.0995 .

\section{1-((2-苯基烯丙氧基)甲基)苯 (1f $)^{[24]}$}

化合物 1f 采用与 1e 相同的方法, 以 2-苯基烯丙 醇, $\mathrm{NaH}$ 和 $\mathrm{BnCl}$ 为原料合成. 无色油状物, 收率 $85 \%$. ${ }^{1} \mathrm{H}$ NMR $\left(400 \mathrm{MHz}, \mathrm{CDCl}_{3}\right) \delta 7.47(\mathrm{~d}, J=8.0 \mathrm{~Hz}, 2 \mathrm{H})$, $7.36 \sim 7.29(\mathrm{~m}, 8 \mathrm{H}), 5.56(\mathrm{~s}, 1 \mathrm{H}), 5.38(\mathrm{~s}, 1 \mathrm{H}), 4.57(\mathrm{~s}$, $2 \mathrm{H}), 4.42$ (s, 2H).

\section{3 镍催化不对称烯氢化反应典型操作}

在手套箱中, 将手性磷配体 $(0.02 \mathrm{mmol})$ 和 $[\mathrm{Ni}(\text { allyl }) \mathrm{Br}]_{2}(0.01 \mathrm{mmol})$ 称于 Schlenk 管 $\mathrm{A}$ 中; 将 $\operatorname{NaBAr}_{\mathrm{F}}(0.024 \mathrm{mmol})$ 称于 Schlenk 管 B 中. 取出后, 向 $\mathrm{A}$ 管加入 $2.5 \mathrm{~mL}$ 二氯甲烷, 络合 $1 \mathrm{~min}$, 将 $\mathrm{A}$ 中 的溶液用注射器转移到 $\mathrm{B}$ 管中, 络合 $3 \mathrm{~min}$, 然后将 此 B 管放入恒温槽中, 迅速将 B 管中的气体置换成乙 烯 (3 次), 在乙烯气氛下向体系滴加三甲基(2-芳基 烯丙氧基)硅烷底物 $(0.5 \mathrm{mmol})$, 在 $1 \mathrm{~min}$ 内滴完. 用 气相色谱检测反应的转化率和选择性. 待反应完毕 后, 放掉乙烯气, 蒸去二氯甲烷, 加入 $5 \mathrm{~mL}$ 干燥的 THF 和 $50 \mathrm{mg}$ 四丁基氟化铵, 室温搅拌脱去保护基. 将反应体系浓缩后经硅胶柱层析得到脱保护基的烯 氢化产物 $\mathbf{4}$, 其分析数据如下.

(2R)-2-甲基-2-苯基丁-3-烯-1-醇 $(\mathbf{4 c})^{[25]}$

无色油状物, 收率 77\%, $81 \% \mathrm{ee},[\alpha]_{\mathrm{D}}{ }^{20}=-18.1(\mathrm{c}$ 1.9, EtOH). (-)-4c 的绝对构型为 $R$ (参考化合物 $(S)-\mathbf{6}$ 确定). SFC 条件: Chiralcel OJ-H 柱, $\mathrm{sc} \mathrm{CO}_{2} / i-\mathrm{PrOH}=$ 90:10, 流速 $=2.0 \mathrm{~mL} / \mathrm{min}$, 检测器波长 $=220 \mathrm{~nm}$, 压力 $=100 \mathrm{bar}, t_{\mathrm{R}}=5.19 \mathrm{~min}$ 对应 $S$ 异构体, $t_{\mathrm{R}}=5.58$ min 对应 $R$ 异构体. ${ }^{1} \mathrm{H}$ NMR $\left(300 \mathrm{MHz}, \mathrm{CDCl}_{3}\right) \delta$ 7.35-7.30 (m, 4H), 7.25 7.21 (m, 1H), $6.07(\mathrm{dd}, J=$ 17.7 and $11.1 \mathrm{~Hz}, 1 \mathrm{H}), 5.26(\mathrm{dd}, J=10.8$ and $1.2 \mathrm{~Hz}$, $1 \mathrm{H}), 5.15(\mathrm{dd}, J=17.7$ and $1.2 \mathrm{~Hz}, 1 \mathrm{H}), 3.78(\mathrm{~d}, J=3.6$ $\mathrm{Hz}, 2 \mathrm{H}), 1.46$ (s, 1H), 1.42 (s, 3H).

(2R)-2-甲基-2-对甲基苯基丁-3-烯-1-醇 $(\mathbf{4 g})^{[26]}$

无色油状物, 收率 $91 \%, 78 \% \mathrm{ee},[\alpha]_{\mathrm{D}}{ }^{20}=-10.4(c$ $\left.1.25, \mathrm{CHCl}_{3}\right)$. 通过和文献比旋光对照确定 (-) $-\mathbf{4 g}$ 的 绝对构型为 $R$ [文献: $(R)$-异构体 $[\alpha]_{\mathrm{D}}{ }^{20}=-2.4$ (c 1.2, $\left.\left.\mathrm{CHCl}_{3}\right)\right]^{[26]}$. SFC 条件: Chiralcel OJ-H 柱, sc $\mathrm{CO}_{2} / i-\mathrm{PrOH}=90: 10$, 流速 $=2.0 \mathrm{~mL} / \mathrm{min}$, 检测器波 长 $=220 \mathrm{~nm}$, 压力 $=100 \mathrm{bar}, t_{\mathrm{R}}=4.89 \mathrm{~min}$ 对应 $S$ 异 构体, $t_{\mathrm{R}}=5.48 \mathrm{~min}$ 对应 $R$ 异构体. ${ }^{1} \mathrm{H}$ NMR $(300 \mathrm{MHz}$, $\left.\mathrm{CDCl}_{3}\right) \delta 7.24(\mathrm{~d}, J=8.1 \mathrm{~Hz}, 2 \mathrm{H}), 7.15(\mathrm{~d}, J=8.1 \mathrm{~Hz}$, 2H), $6.07(\mathrm{dd}, J=17.7$ and $10.8 \mathrm{~Hz}, 1 \mathrm{H}), 5.25(\mathrm{dd}, J=$ 10.8 and $0.9 \mathrm{~Hz}, 1 \mathrm{H}), 5.14(\mathrm{dd}, J=17.7$ and $0.9 \mathrm{~Hz}$, $1 \mathrm{H}), 3.77(\mathrm{~d}, J=5.7 \mathrm{~Hz}, 2 \mathrm{H}), 2.33(\mathrm{~s}, 3 \mathrm{H}), 1.55(\mathrm{~s}, 1 \mathrm{H})$, $1.41(\mathrm{~s}, 3 \mathrm{H})$.

2-甲基-2-(4-甲氧基苯基)丁-3-烯-1-醇 $(\mathbf{4 h})^{[27]}$

无色油状物, 收率 $97 \%, 85 \% \mathrm{ee},[\alpha]_{\mathrm{D}}{ }^{20}=-21.0(\mathrm{c}$ $1.5, \mathrm{EtOH}) . \mathrm{SFC}$ 条件: Chiralcel OJ-H 柱, sc $\mathrm{CO}_{2} / i-\mathrm{PrOH}=90: 10$, 流速 $=2.0 \mathrm{~mL} / \mathrm{min}$, 检测器波 长 $=220 \mathrm{~nm}$, 压力 $=100 \mathrm{bar}, t_{\mathrm{R}}=6.56 \mathrm{~min}$ 对应小峰, $t_{\mathrm{R}}=7.08 \mathrm{~min}$ 对应大峰. ${ }^{1} \mathrm{H} \mathrm{NMR}\left(300 \mathrm{MHz}, \mathrm{CDCl}_{3}\right) \delta$ $7.26(\mathrm{~d}, J=12.0 \mathrm{~Hz}, 2 \mathrm{H}), 6.87(\mathrm{~d}, J=11.7 \mathrm{~Hz}, 2 \mathrm{H})$, $6.05(\mathrm{dd}, J=17.7$ and $10.8 \mathrm{~Hz}, 1 \mathrm{H}), 5.23(\mathrm{dd}, J=10.8$ and $1.2 \mathrm{~Hz}, 1 \mathrm{H}), 5.12(\mathrm{dd}, J=17.7$ and $1.2 \mathrm{~Hz}, 1 \mathrm{H})$, 3.79 (s, 3H), 3.74 (s, 2H), 1.45 (s, 1H), 1.40 (s, 3H).

2-甲基-2-(4-氟苯基)丁-3-烯-1-醇 (4i)

无色油状物, 收率 $80 \%, 94 \% \mathrm{ee},[\alpha]_{\mathrm{D}}{ }^{20}=-16.7(c$ 1.6, EtOH). HPLC 条件: Chiralcel OJ-H 柱, 正己烷 / 异丙醇 $=95: 5$, 流速 $=0.8 \mathrm{~mL} / \mathrm{min}$, 检测器波长 $=$ $220 \mathrm{~nm}, t_{\mathrm{R}}=22.22 \mathrm{~min}$ 对应大峰, $t_{\mathrm{R}}=24.17 \mathrm{~min}$ 对应 小峰. ${ }^{1} \mathrm{H}$ NMR (300 MHz, $\left.\mathrm{CDCl}_{3}\right) \delta 7.23$ (dd, $J=8.7$ and $5.4 \mathrm{~Hz}, 2 \mathrm{H}), 6.93(\mathrm{t}, J=8.4 \mathrm{~Hz}, 2 \mathrm{H}), 5.95(\mathrm{dd}, J=$ 17.7 and $11.1 \mathrm{~Hz}, 1 \mathrm{H}), 5.18(\mathrm{~d}, J=10.5 \mathrm{~Hz}, 1 \mathrm{H}), 5.04$ $(\mathrm{d}, J=18.0 \mathrm{~Hz}, 1 \mathrm{H}), 3.67(\mathrm{~s}, 2 \mathrm{H}), 1.45(\mathrm{~s}, 1 \mathrm{H}), 1.32(\mathrm{~s}$, $3 \mathrm{H}) ;{ }^{13} \mathrm{C}$ NMR $\left(75 \mathrm{MHz}, \mathrm{CDCl}_{3}\right) \delta 163.1,159.9,143.5$, 140.3, 140.2, 128.6, 128.5, 115.2, 115.0, 114.7, 70.0, 46.5, 22.8; HRMS (EI)计算值 $\mathrm{C}_{11} \mathrm{H}_{13} \mathrm{FO}: 180.0950$; 实 测值 180.0950 .

2-甲基-2-(4-氯苯基)丁-3-烯-1-醇 (4j)

无色油状物, 收率 $69 \%, 92 \% \mathrm{ee},[\alpha]_{\mathrm{D}}{ }^{20}=-20.6(c$ 0.65, EtOH). HPLC 条件: Chiralcel OJ-H 柱, 正己烷/ 
异丙醇 $=97: 3$, 流速 $=0.8 \mathrm{~mL} / \mathrm{min}$, 检测器波长 $=$ $220 \mathrm{~nm}, t_{\mathrm{R}}=23.32 \mathrm{~min}$ 对应大峰, $t_{\mathrm{R}}=24.79 \mathrm{~min}$ 对应 小峰. ${ }^{1} \mathrm{H}$ NMR $\left(400 \mathrm{MHz}, \mathrm{CDCl}_{3}\right) \delta 7.29(\mathrm{~d}, J=1.6 \mathrm{~Hz}$, $4 \mathrm{H}), 6.03(\mathrm{dd}, J=17.6$ and $10.8 \mathrm{~Hz}, 1 \mathrm{H}), 5.28(\mathrm{~d}, J=$ $10.8 \mathrm{~Hz}, 1 \mathrm{H}), 5.14(\mathrm{~d}, J=17.6 \mathrm{~Hz}, 1 \mathrm{H}), 3.76(\mathrm{~s}, 2 \mathrm{H})$, $1.43(\mathrm{~s}, 1 \mathrm{H}), 1.40(\mathrm{~s}, 3 \mathrm{H}) ;{ }^{13} \mathrm{C} \mathrm{NMR}\left(75 \mathrm{MHz}, \mathrm{CDCl}_{3}\right) \delta$ 143.1, 132.4, 128.5, 128.4, 115.1, 69.8, 46.7, 22.7; HRMS (EI) 计算值 $\mathrm{C}_{11} \mathrm{H}_{13} \mathrm{ClO}: 196.0655$; 实测值 196.0654.

2-甲基-2-(4-澳苯基)丁-3-烯-1-醇 (4k)

无色油状物，收率 $62 \%, 95 \% \mathrm{ee},[\alpha]_{\mathrm{D}}{ }^{20}=-20.1$ (c 1.0, EtOH). HPLC 条件: Chiralcel OJ-H 柱, 正己烷 /异丙醇 $=98: 2$, 流速 $=0.7 \mathrm{~mL} / \mathrm{min}$, 检测器波长 $=$ $220 \mathrm{~nm}, t_{\mathrm{R}}=25.97 \mathrm{~min}$ 对应大峰, $t_{\mathrm{R}}=27.59 \mathrm{~min}$ 对应 小峰. ${ }^{1} \mathrm{H}$ NMR $\left(400 \mathrm{MHz}, \mathrm{CDCl}_{3}\right) \delta 7.44(\mathrm{~d}, J=8.4$ $\mathrm{Hz}, 2 \mathrm{H}), 7.21(\mathrm{~d}, J=8.4 \mathrm{~Hz}, 2 \mathrm{H}), 6.01(\mathrm{dd}, J=18.0$ and $10.8 \mathrm{~Hz}, 1 \mathrm{H}), 5.26(\mathrm{~d}, J=10.8 \mathrm{~Hz}, 1 \mathrm{H}), 5.12(\mathrm{~d}, J$ $=17.6 \mathrm{~Hz}, 1 \mathrm{H}), 3.74(\mathrm{~s}, 2 \mathrm{H}), 1.45(\mathrm{~s}, 1 \mathrm{H}), 1.38(\mathrm{~s}, 3 \mathrm{H})$; ${ }^{13} \mathrm{C}$ NMR $\left(100 \mathrm{MHz}, \mathrm{CDCl}_{3}\right) \delta 143.9,143.3,131.7$, 129.1, 120.7, 115.3, 70.0, 47.0, 22.9; HRMS (EI) 计算 值 $\mathrm{C}_{11} \mathrm{H}_{13} \mathrm{BrO}: 240.0150$; 实测值 240.0150 .

2-甲基-2-(4-联苯基)丁-3-烯-1-醇 (4I)

白色固体 (mp: $\left.43-45{ }^{\circ} \mathrm{C}\right)$, 收率 82\%, 80\% ee, $[\alpha]_{\mathrm{D}}{ }^{20}=-26.6$ (c 1.35, EtOH). SFC 条件: Chiralcel OD-H 柱, $\mathrm{sc} \mathrm{CO}_{2} / i-\mathrm{PrOH}=80: 20$, 流速 $=2.0 \mathrm{~mL} / \mathrm{min}$, 检测器波长 $=254 \mathrm{~nm}$, 压力 $=100 \mathrm{bar}, t_{\mathrm{R}}=7.19 \mathrm{~min}$ 对应大峰, $t_{\mathrm{R}}=8.02 \mathrm{~min}$ 对应小峰. ${ }^{1} \mathrm{H}$ NMR $(400 \mathrm{MHz}$, $\left.\mathrm{CDCl}_{3}\right) \delta 7.59 \sim 7.33(\mathrm{~m}, 9 \mathrm{H}), 6.11$ (dd, $J=17.6$ and $10.8 \mathrm{~Hz}, 1 \mathrm{H}), 5.31(\mathrm{~d}, J=10.8 \mathrm{~Hz}, 1 \mathrm{H}), 5.20(\mathrm{~d}, J=$ $17.6 \mathrm{~Hz}, 1 \mathrm{H}), 3.84(\mathrm{~d}, J=6.4 \mathrm{~Hz}, 2 \mathrm{H}), 1.57(\mathrm{~s}, 1 \mathrm{H})$, 1.47 (s, 3H); ${ }^{13} \mathrm{C}$ NMR $\left(75 \mathrm{MHz}, \mathrm{CDCl}_{3}\right) \delta 143.7,143.6$, $140.8,139.4,128.9,127.4,127.3,127.2,127.1,114.7$, 70.1，46.8，22.7; HRMS (EI) 计算值 $\mathrm{C}_{17} \mathrm{H}_{18} \mathrm{O}$ : 238.1358; 实测值 238.1358 .

\section{2-甲基-2-(3-甲氧基苯基)丁-3-烯-1-醇 (4m)}

无色油状物，收率 $86 \%, 76 \%$ ee, $[\alpha]_{\mathrm{D}}{ }^{20}=-17.2(c$ 2.25, EtOH). HPLC 条件: Chiralcel OJ-H 柱, 正已烷/ 异丙醇 $=90: 10$, 流速 $=0.8 \mathrm{~mL} / \mathrm{min}$, 检测器波长 $=$ $220 \mathrm{~nm}, t_{\mathrm{R}}=19.57 \mathrm{~min}$ 对应小峰, $t_{\mathrm{R}}=21.20 \mathrm{~min}$ 对应 大峰. ${ }^{1} \mathrm{H}$ NMR $\left(300 \mathrm{MHz}, \mathrm{CDCl}_{3}\right) \delta 7.18(\mathrm{t}, J=7.8 \mathrm{~Hz}$, $1 \mathrm{H}), 6.87 \sim 6.68(\mathrm{~m}, 3 \mathrm{H}), 5.98(\mathrm{dd}, J=17.4$ and $10.8 \mathrm{~Hz}$, 1H), $5.17(\mathrm{~d}, J=10.8 \mathrm{~Hz}, 1 \mathrm{H}), 5.07(\mathrm{~d}, J=17.4 \mathrm{~Hz}$, 1H), $3.72(\mathrm{~s}, 3 \mathrm{H}), 3.68(\mathrm{~s}, 2 \mathrm{H}), 1.43(\mathrm{~s}, 1 \mathrm{H}), 1.33(\mathrm{~s}$,
$3 \mathrm{H}) ;{ }^{13} \mathrm{C}$ NMR $\left(100 \mathrm{MHz}, \mathrm{CDCl}_{3}\right) \delta 159.9,146.5,143.7$, 129.6, 119.4, 114.7, 113.8, 111.5, 70.2, 55.4, 47.2, 22.8; HRMS (EI) 计算值 $\mathrm{C}_{12} \mathrm{H}_{16} \mathrm{O}_{2}: 192.1150$; 实测 值 192.1150 .

\section{2-甲基-2-(3-氟苯基)丁-3-烯-1-醇（4n)}

无色油状物, 收率 $49 \%, 91 \% \mathrm{ee},[\alpha]_{\mathrm{D}}{ }^{20}=-17.5(c$ $0.85, \mathrm{EtOH})$. SFC 条件: Chiralpak AD-H 柱, sc $\mathrm{CO}_{2} / i-\mathrm{PrOH}=95: 5$, 流速 $=2.0 \mathrm{~mL} / \mathrm{min}$, 检测器波长 $=220 \mathrm{~nm}$, 压力 $=100 \mathrm{bar}, t_{\mathrm{R}}=7.55 \mathrm{~min}$ 对应小峰, $t_{\mathrm{R}}$ $=8.54 \mathrm{~min}$ 对应大峰. ${ }^{1} \mathrm{H}$ NMR $\left(300 \mathrm{MHz}, \mathrm{CDCl}_{3}\right) \delta$ $7.27 \sim 7.19(\mathrm{~m}, 1 \mathrm{H}), 7.07 \sim 6.97(\mathrm{~m}, 2 \mathrm{H}), 6.86(\mathrm{t}, J=8.1$ $\mathrm{Hz} 1 \mathrm{H}), 5.97(\mathrm{dd}, J=17.7$ and $10.8 \mathrm{~Hz}, 1 \mathrm{H}), 5.21(\mathrm{~d}, J$ $=10.8 \mathrm{~Hz}, 1 \mathrm{H}), 5.08(\mathrm{~d}, J=17.7 \mathrm{~Hz}, 1 \mathrm{H}), 3.70(\mathrm{~s}, 2 \mathrm{H})$, $1.34(\mathrm{~s}, 3 \mathrm{H}), 1.32(\mathrm{~s}, 1 \mathrm{H}) ;{ }^{13} \mathrm{C}$ NMR $\left(75 \mathrm{MHz}, \mathrm{CDCl}_{3}\right)$ $\delta 164.6,161.3,143.0,129.9,129.7,122.5,115.1,114.4$, 114.1, 113.5, 113.2, 69.8, 47.0, 22.6; HRMS (EI) 计算 值 $\mathrm{C}_{11} \mathrm{H}_{13} \mathrm{FO}$ : 180.0950 ; 实测值 180.0955 .

2-甲基-2-(3-氯苯基)丁-3-烯-1-醇 (4o)

无色油状物, 收率 $42 \%, 88 \%$ ee, $[\alpha]_{\mathrm{D}}{ }^{20}=-15.7$ ( $c$ 0.75, EtOH). SFC 条件: Chiralpak AD-H 柱, sc $\mathrm{CO}_{2} / i-\mathrm{PrOH}=95: 5$, 流速 $=2.0 \mathrm{~mL} / \mathrm{min}$, 检测器波长 $=220 \mathrm{~nm}$, 压力 $=100 \mathrm{bar}, t_{\mathrm{R}}=11.32 \mathrm{~min}$ 对应小峰, $t_{\mathrm{R}}$ $=12.27$ min 对应大峰. ${ }^{1} \mathrm{H}$ NMR $\left(400 \mathrm{MHz}, \mathrm{CDCl}_{3}\right) \delta$ $7.33 \sim 7.22(\mathrm{~m}, 4 \mathrm{H}), 6.03(\mathrm{dd}, J=17.6$ and $10.8 \mathrm{~Hz}, 1 \mathrm{H})$, $5.29(\mathrm{~d}, J=10.8 \mathrm{~Hz}, 1 \mathrm{H}), 5.16(\mathrm{~d}, J=17.6 \mathrm{~Hz}, 1 \mathrm{H})$, $3.77(\mathrm{~s}, 2 \mathrm{H}), 1.48(\mathrm{~s}, 1 \mathrm{H}), 1.41(\mathrm{~s}, 3 \mathrm{H}) ;{ }^{13} \mathrm{C}$ NMR $(75$ $\left.\mathrm{MHz}, \mathrm{CDCl}_{3}\right) \delta 146.9,142.9,134.4,129.6,127.4,126.7$, 125.2, 125.1, 115.2, 69.8, 47.0, 22.6; HRMS (EI) 计算 值 $\mathrm{C}_{11} \mathrm{H}_{13} \mathrm{ClO}$ : 196.0655; 实测值 196.0654.

2-甲基-2-(3-澳苯基)丁-3-烯-1-醇 (4p)

无色油状物, 收率 $49 \%, 85 \% \mathrm{ee},[\alpha]_{\mathrm{D}}{ }^{20}=-14.0(c$ 1.85, EtOH) SFC 条件: Chiralpak AD-H 柱, sc $\mathrm{CO}_{2} / i-\mathrm{PrOH}=95: 5$, 流速 $=1.0 \mathrm{~mL} / \mathrm{min}$, 检测器波长 $=220 \mathrm{~nm}$, 压力 $=100 \mathrm{bar}, t_{\mathrm{R}}=30.64 \mathrm{~min}$ 对应小峰, $t_{\mathrm{R}}=32.77 \mathrm{~min}$ 对应大峰. ${ }^{1} \mathrm{H}$ NMR $\left(400 \mathrm{MHz}, \mathrm{CDCl}_{3}\right) \delta$ $7.49 \sim 7.19(\mathrm{~m}, 4 \mathrm{H}), 6.02(\mathrm{dd}, J=17.6$ and $10.8 \mathrm{~Hz}, 1 \mathrm{H})$, $5.29(\mathrm{dd}, J=10.8$ and $0.8 \mathrm{~Hz}, 1 \mathrm{H}), 5.16(\mathrm{dd}, J=17.6$ and $0.8 \mathrm{~Hz}, 1 \mathrm{H}), 3.76(\mathrm{~d}, J=5.2 \mathrm{~Hz}, 2 \mathrm{H}), 1.47(\mathrm{~s}, 1 \mathrm{H})$, $1.40(\mathrm{~s}, 3 \mathrm{H}) ;{ }^{13} \mathrm{C}$ NMR $\left(75 \mathrm{MHz}, \mathrm{CDCl}_{3}\right) \delta 147.1,142.9$, $130.3,130.0,129.7,125.6,122.7,115.3,69.8,47.0$, 22.6; HRMS (EI) 计算值 $\mathrm{C}_{11} \mathrm{H}_{13} \mathrm{BrO}$ : 240.0150 ; 实测 值 240.0150 .

2-甲基-2-(苯并 $[1,3]$ 二氧环戊烷-5-基)丁-3-烯-1- 


\section{醇 $(\mathbf{4 q})$}

无色油状物，收率 $92 \%, 89 \% \mathrm{ee},[\alpha]_{\mathrm{D}}{ }^{20}=-22.0(c$ 0.5, EtOH). HPLC 条件: Chiralcel OJ-H 柱, 正已烷/异 丙醇 $=90: 10$, 流速 $=0.8 \mathrm{~mL} / \mathrm{min}$, 检测器波长 $=$ $220 \mathrm{~nm}, t_{\mathrm{R}}=21.64 \mathrm{~min}$ 对应小峰, $t_{\mathrm{R}}=22.88 \mathrm{~min}$ 对应 大峰. ${ }^{1} \mathrm{H}$ NMR $\left(300 \mathrm{MHz}, \mathrm{CDCl}_{3}\right) \delta 6.77 \sim 6.66(\mathrm{~m}, 3 \mathrm{H})$, $5.93(\mathrm{dd}, J=17.7$ and $10.8 \mathrm{~Hz}, 1 \mathrm{H}), 5.83(\mathrm{~s}, 2 \mathrm{H}), 5.14$ (d, $J=10.8 \mathrm{~Hz}, 1 \mathrm{H}), 5.03(\mathrm{~d}, J=17.7 \mathrm{~Hz}, 1 \mathrm{H}), 3.62(\mathrm{~s}$, 2H), $1.61(\mathrm{~s}, 1 \mathrm{H}), 1.28(\mathrm{~s}, 3 \mathrm{H}) ;{ }^{13} \mathrm{C}$ NMR $(75 \mathrm{MHz}$, $\left.\mathrm{CDCl}_{3}\right) \delta 147.8,146.0,143.7,138.5,120.0,114.3$, 108.0, 107.8, 101.0, 70.1, 46.7, 22.9; HRMS (EI) 计算 值 $\mathrm{C}_{12} \mathrm{H}_{14} \mathrm{O}_{3}$ : 206.0943; 实测值 206.0945.

2-甲基-2-(2-荎基)丁-3-烯-1-醇 (4r)

灰白色固体(mp: 40 42 ${ }^{\circ} \mathrm{C}$ ), 收率 $92 \%, 81 \%$ ee, $[\alpha]_{\mathrm{D}}{ }^{20}=-29.0(c 1.0, \mathrm{EtOH})$. 将 $\mathbf{4 r}$ 转化为相应的苯甲 酸酯测定其 ee 值. HPLC 条件: Chiralcel OJ 柱, 正己烷 /异丙醇 $=90: 10$, 流速 $=0.8 \mathrm{~mL} / \mathrm{min}$, 检测器波长 $=$ $254 \mathrm{~nm}, t_{\mathrm{R}}=12.73 \mathrm{~min}$ 对应小峰, $t_{\mathrm{R}}=28.63 \mathrm{~min}$ 对应大 峰. ${ }^{1} \mathrm{H}$ NMR $\left(300 \mathrm{MHz}, \mathrm{CDCl}_{3}\right) \delta$ 7.68 7.65 (m, 4H), $7.35 \sim 7.31(\mathrm{~m}, 3 \mathrm{H}), 6.00(\mathrm{dd}, J=17.7$ and $10.8 \mathrm{~Hz}, 1 \mathrm{H})$, $5.16(\mathrm{~d}, J=10.8 \mathrm{~Hz}, 1 \mathrm{H}), 5.03(\mathrm{~d}, J=17.7 \mathrm{~Hz}, 1 \mathrm{H}), 3.70$ (s, 2H), $1.55(\mathrm{~s}, 1 \mathrm{H}), 1.37(\mathrm{~s}, 3 \mathrm{H}) ;{ }^{13} \mathrm{C}$ NMR $(75 \mathrm{MHz}$, $\left.\mathrm{CDCl}_{3}\right) \delta 143.7,142.0,133.5,132.2,128.1,127.5,126.1$,
125.9, 125.7 125.5, 114.9, 69.9, 47.2, 22.8; HRMS (EI) 计算值 $\mathrm{C}_{15} \mathrm{H}_{16} \mathrm{O}: 212.1201$; 实测值 212.1206.

2-甲基-2-(2-噻吩基)丁-3-烯-1-醇 (4s)

无色油状物，收率 $90 \%, 73 \% \mathrm{ee},[\alpha]_{\mathrm{D}}{ }^{20}=-26.6(c$ 1.7, EtOH). SFC 条件: Chiralcel OJ-H 柱, sc $\mathrm{CO}_{2} / i-\mathrm{PrOH}=95: 5$, 流速 $=2.0 \mathrm{~mL} / \mathrm{min}$, 检测器波长 $=220 \mathrm{~nm}$, 压力 $=100 \mathrm{bar}, t_{\mathrm{R}}=23.11 \mathrm{~min}$ 对应小峰, $t_{\mathrm{R}}$ $=24.20 \mathrm{~min}$ 对应大峰. ${ }^{1} \mathrm{H}$ NMR $\left(300 \mathrm{MHz}, \mathrm{CDCl}_{3}\right) \delta$ $7.11(\mathrm{~d}, J=4.8 \mathrm{~Hz}, 1 \mathrm{H}), 6.90 \sim 6.81(\mathrm{~m}, 2 \mathrm{H}), 6.01$ (dd, $J$ $=17.4$ and $10.8 \mathrm{~Hz}, 1 \mathrm{H}), 5.14(\mathrm{~d}, J=10.8 \mathrm{~Hz}, 1 \mathrm{H}), 5.07$ (d, $J=17.4 \mathrm{~Hz}, 1 \mathrm{H}), 3.61$ (s, 2H), $1.79(\mathrm{~s}, 1 \mathrm{H}), 1.41$ (s, $3 \mathrm{H}) ;{ }^{13} \mathrm{C}$ NMR $\left(75 \mathrm{MHz}, \mathrm{CDCl}_{3}\right) \delta 149.5,142.9,126.8$, 124.0，114.7，71.1，45.9，23.5; HRMS (EI) 计算值 $\mathrm{C}_{9} \mathrm{H}_{12} \mathrm{OS}$ : 168.0609; 实测值 168.0604 .

\section{$4.4(S)-\alpha$-甲基莨宕酸(6)的合成}

$(S)$ - $\alpha$-甲基莨若酸按照此前报道的方法 ${ }^{[14]}$, 从光 学活性 (2R)-2-甲基-2-苯基丁-3-烯-1-醇 (4c) 出发合 成. 收率 77\%, 暗棕色油状物. $[\alpha]_{\mathrm{D}}{ }^{20}=-19.0$ (c 1.0, $\mathrm{CHCl}_{3}$ ) (文献值 ${ }^{[28]}$ : $[\alpha]_{\mathrm{D}}{ }^{25}=-19.4\left(c 1.65, \mathrm{CHCl}_{3}\right)$ 对 应 $S$-富集的 6); ${ }^{1} \mathrm{H}$ NMR $\left(400 \mathrm{MHz}, \mathrm{CDCl}_{3}\right) \delta$ 7.35-7.30 (m, 5H), $4.10(\mathrm{~d}, J=11.6 \mathrm{~Hz}, 1 \mathrm{H}), 3.65(\mathrm{~d}, J$ $=11.2 \mathrm{~Hz}, 1 \mathrm{H}), 1.68(\mathrm{~s}, 3 \mathrm{H})$.

\section{参考文献}

1 Jolly PW, Wilke G. in Applied Homogeneous Catalysis with Organometallic Compounds, Vol 2. (Eds. Cornils B, Herrmann WA), New York: Wiley-VCH, 1996. 1024-1048

2 Goossen LJ. Asymmetric hydrovinylation: New perspectives through use of modular ligand systems. Angew Chem Int Ed, 2002, 41: $3775-3778$

3 RajanBabu TV. Asymmetric hydrovinylation reaction. Chem Rev, 2003, 103: 2845-2860

4 Wilke G, Monkiewicz J. DOS 3618 169, priority 30.05.1986; Chem Abstr, 1988, 109: P6735

5 Park H, RajanBabu TV. Tunable ligands for asymmetric catalysis: Readily available carbohydrate-derived diarylphosphinites induce high selectivity in the hydrovinylation of styrene derivatives. J Am Chem Soc, 2002, 124: 734-735

6 Franciò G, Faraone F, Leitner W. Highly enantioselective nickel-catalyzed hydrovinylation with chiral phosphoramidite ligands. J Am Chem Soc, 2002, 124: 736-737

7 Zhang A, RajanBabu TV. Fine-tuning monophosphine ligands for enhanced enantioselectivity. Influence of chiral hemilabile pendant groups. Org Lett, 2004, 6: 1515-1517

8 Smith CR, RajanBabu TV. Efficient, selective, and green: Catalyst tuning for highly enantioselective reactions of ethylene. Org Lett, 2008, 10: $1657-1659$

9 Shi W-J, Zhang Q, Xie J-H, Zhu S-F, Hou G-H, Zhou Q-L. Highly enantioselective hydrovinylation of $\alpha$-alkyl vinylarenes. An approach to the construction of all-carbon quaternary stereocenters. J Am Chem Soc, 2006, 128: 2780-2781 
10 Zhang A, RajanBabu TV. All-carbon quaternary centers via catalytic asymmetric hydrovinylation. New approaches to the exocyclic side chain stereochemistry problem. J Am Chem Soc, 2006, 128: 5620-5621

11 Kumareswaran R, Nandi M, RajanBabu TV. Hydrovinylation of norbornene. Ligand-dependent selectivity and asymmetric variations. Org Lett, 2003, 5: 4345-4348

12 Zhang A, RajanBabu TV. Hydrovinylation of 1,3-dienes: A new protocol, an asymmetric variation, and a potential solution to the exocyclic side chain stereochemistry problem. J Am Chem Soc, 2006, 128: 54-55

13 Saha B, Smith CR, RajanBabu TV. Ligand tuning in asymmetric hydrovinylation of 1,3-dienes: A stereoselective route to either steroid- $\mathrm{C}_{20}(S)$ or $-\mathrm{C}_{20}(R)$ derivatives. $J$ Am Chem Soc, 2008, 130: 9000-9005

14 Zhang Q, Zhu S-F, Qiao X-C, Wang L-X, Zhou Q-L. Nickel-catalyzed highly selective hydrovinylation of $\alpha$-ketals of vinylarenes. Adv Synth Catal, 2008, 350: 1507-1510

15 Xie J-H, Zhou Q-L. Chiral diphosphine and monodentate phosphorous ligands on a spiro scaffold for transition-metal-catalyzed asymmetric reactions. Acc Chem Res, 2008, 41: 581-593

16 Melone G, Vecchi A, Pagani G, Testa E. Tropine DL- $\alpha$-methyltropate (methyltropine) and its optical antipodes. J Org Chem, 1960, 25: $859-861$

17 Wilke G. $\pi$-Allyl nickel compounds and their preparation. US3422128, 1969

18 Nishida H, Takada N, Yoshimura M, Sonoda T, Kobayashi H. Tetrakis[3,5-bis(trifluoromethyl)phenyl]borate. Highly lipophilic stable anionic agent for solvent-extraction cations. Bull Chem Soc Jpn, 1984, 57: 2600-2604

19 Brookhart M, Grant B, Volpe AF Jr. [(3,5-( $\left.\left.\left(\mathrm{CF}_{3}\right)_{2} \mathrm{C}_{6} \mathrm{H}_{3}\right)_{4} \mathrm{~B}\right]^{-}\left[\mathrm{H}\left(\mathrm{OEt}_{2}\right)_{2}\right]^{+}$: A convenient reagent for generation and stabilization of cationic, highly electrophilic organometallic complexes. Organometallics, 1992, 11: 3920-3922

20 Zhou H, Wang W-H, Fu Y, Xie J-H, Shi W-J, Wang L-X, Zhou Q-L. Highly enantioselective copper-catalyzed conjugate addition of diethylzinc to enones using chiral spiro phosphoramidites as ligands. J Org Chem, 2003, 68: 1582-1584

21 Shi W-J, Wang L-X, Fu Y, Zhu S-F, Zhou Q-L. Highly regioselective asymmetric copper-catalyzed allylic alkylation with dialkylzinc using monodentate chiral spiro phosphoramidite and phosphite ligands. Tetrahedron: Asymmetry, 2003, 14: 3867-3872

22 Yang Y, Zhu S-F, Duan H-F, Zhou C-Y, Wang L-X, Zhou Q-L. Asymmetric reductive coupling of dienes and aldehydes catalyzed by nickel complexes of spiro phosphoramidites: highly enantioselective synthesis of chiral bishomoallylic alcohols. J Am Chem Soc, 2007, 129: $2248-2249$

23 Duboudin JG, Jousseaume B, Saux A. Reactifs de grignard vinyliques $\gamma$ fonctionnels: I. Reactivite des organomagnesiens vis-a-vis d'alcools $\alpha$ acetyleniques en presence d'halogenures cuivreux. J Organomet Chem 1979, 168: 1-11

24 Barluenga J, Fañanás FJ, Sanz R, Marcos C, Ignacio JM. 2-Arylallyl as a new protecting group for amines, amides and alcohols. Chem Commun, 2005, 933-935

25 Tueting DR, Echavarren AM, Stille JK. Palladium catalyzed coupling of organostannanes with vinyl epoxides. Tetrahedron, 1989, 45: 979-992

26 Fadel A, Canet JL, Salaün J. Asymmetric construction of quaternary carbons from chiral malonates: Total synthesis of (+)-epilaurene and (-)-isolaurene. Tetrahedron: Asymmetry, 1993, 4: 27-30

27 Fadel A, Vandromme L. Total synthesis of (-)-sporochnol A, the fish deterrent from a chiral malonate. Tetrahedron: Asymmetry, 1999, 10: $1153-1162$

28 Imao D, Itoi A, Yamazaki A, Shirakura M, Ohtoshi R, Ogata K, Ohmori Y, Ohta T, Ito Y. Easy access to esters with a benzylic quaternary carbon center from diallyl malonates by palladium-catalyzed decarboxylative allylation. J Org Chem, 2007, 72, 1652-1658 


\title{
Nickel-catalyzed enantioselective hydrovinylation of silyl-protected allylic alcohols: An efficient access to homoallylic alcohols with a chiral quaternary center
}

ZHANG Qi, ZHU Shou-Fei, CAI Yan, WANG Li-Xin \& ZHOU Qi-Lin

State Key Laboratory and Institute of Elemento-organic Chemistry; Nankai University, Tianjin 300071, China

\begin{abstract}
An asymmetric hydrovinylation of silyl-protected allylic alcohols catalyzed by nickel complexes of chiral spiro phosphoramidite ligands was developed. A series homoallylic alchols with a chiral quaternary center were produced in high yields (up to 97\%) and high enantioselectivities (up to $95 \%$ ee). The reaction provided an efficient method for preparing bifunctional compounds with a chiral quaternary carbon center.
\end{abstract}

Keywords: asymmetric hydrovinylation, chiral spiro phosphorus ligands, functionalized olefins, chiral quaternary center 